\section{Chance and necessity}

\section{Michael Berry}

Regular and Stochastic Motion.

By A.J. Lichtenberg and M.A.

Lieberman.

Springer-Verlag: 1983.

Pp.500. DM 108. \$44.60.

IN RECENT decades the study of classical mechanics has revived, to the point where it is no exaggeration to claim that the subject is in the full flood of a renaissance. The new developments contain the resolution of an apparent contradiction which puzzled many physicists and mathematicians since Newton.

Consider the motion of a particle acted on by forces. Sometimes the motion is predictable ('regular'), as in the Keplerian motion of planets whose mutual gravitation is neglected, and sometimes the motion is unpredictable (chaotic or 'stochastic'), as in a pinball machine. Now we are learning that between these extremes of 'necessity and chance' or 'fate and luck' lie orbit structures with a marvellous richness: for a typical system of forces, some orbits are regular and some are stochastic, depending on initial conditions, and the different types can be intimately mixed like rational numbers among real numbers. Such discoveries must have methodological implications for those sciences which take Newtonian mechanics as their paradigm.

Many research papers have been published and a few general review articles have been written, with the result that the elements of the New Mechanics are becoming widely known. But what has been lacking is a 'workshop manual' in which the techniques are presented in detail from the beginning. This is exactly what Lichtenberg and Lieberman provide and it is very welcome.

The main strength of the book is its comprehensive discussion of how stochasticity grows when a system whose motion is initially regular is subjected to perturbations of increasing strength. Stochas-

\section{Journals review issue 1983}

New week Nature will publish the third review supplement devoted to new science journals. The two previous supplements (covering journals first published between January 1978 and May 1980 and June 1980 and May 1981) appeared in Nature 293, 341-369 (1981) and 299, 491-514 (1982).

Journals appearing between June 1981 and May 1982 and earlier journals not covered in previous issues, have been considered.

ticity sneaks in through resonances, which correspond to periodic orbits of the unperturbed system. The resonances cause classical perturbation theory to be far more complicated than its quantum-mechanical counterpart. Nevertheless, 'superconvergent' schemes show via the celebrated Kolmogorov-Arnold-Moser (KAM) theorem that regular motion does survive perturbation, at least until there is widespread overlap of resonances.

Complicated motion does not require the interaction of many particles (as in a gas) but can occur even for a single particle moving in two dimensions under the action of external forces. For three or more degrees of freedom, however, a qualitatively new feature occurs (the Arnold diffusion) and the thorough discussion of this is another strong point.

The formal developments are abundantly illustrated by examples, whose physical backgrounds are sketched in an appendix. These include: stability of planetary

\section{Boundary matters}

\section{D.H. Everett}

Colloid and Interface Chemistry.

By Robert D. Vold and Marjorie J. Vold. Addison Wesley: 1983. Pp692.

f63.70, $\$ 137.50$.

THERE has long been a need for a comprehensive modern book on colloid and interface chemistry. Kruyt's outstanding $\mathrm{Col}$ loid Science is now over thirty years old, and during this time enormous advances have been made in both the experimental investigation and in the understanding of colloid phenomena.

Robert and Marjorie Vold are among those who have contributed substantially to these advances so that one hoped that their book would fill this important gap in the scientific literature. Alas, in my opinion, that gap still remains. This is a particularly unhappy conclusion, reached reluctantly, as Robert's untimely death prevented him from collaborating in the final stages of publication.

The book is for "everyone interested in surface and colloid science", but mainly for graduate students, with ambitious undergraduates and experienced practitioners as secondary beneficiaries. The breadth and depth of the subject is emphasized by the reply given to the question "How long does it take to master colloid and interface chemistry?" "More than a lifetime.". Perhaps no one, or even a pair of authors, should attempt to cover the whole field. The present authors have attempted, and to some degree succeeded in giving a broad perspective, but the book contains many errors, and the treatment of several subjects is open to serious criticism.

In many instances, a precis of an authoritative article or book is given with motion, particle accelerators, magnetic confinement and chemical dynamics.

I have a few minor criticisms. The important fact that in regular motion the orbits explore tori in the system's phase space is introduced rather late and not emphasized. This leads to a confusion between the concepts of integrability and separability. It is recognized that strange attractors have a hierarchical structure describable in terms of a fractal dimension and yet, unaccountably, the work of Mandelbrot is nowhere mentioned. There is little use of the modern notation of differential forms to simplify phase-space (symplectic) geometry.

In spite of these criticisms I do strongly recommend this book to those who are contemplating or carrying out research in modern mechanics and who need to learn the nuts and bolts of the subject.

Professor Michael Berry is in the H.H. Wills Physics Laboratory, University of Bristol.

the comment that further details are to be found in the original. Too often, these omitted details include some which are absolutely essential to the argument, so that the book cannot be used properly by itself nor understood fully without consultation of the literature cited.

Perhaps the weakest aspect of the book is the thoroughly unsatisfactory use of thermodynamics. The phraseology is loose and definitions are often ambiguous. Adsorption is defined in confusing terms and no justification is given for including surface terms in the equation for the energy of a system containing an interface. How an algebraic manipulation of three equations - none of which includes any surface terms - can lead to an equation including a surface contribution is a mystery. And the identification of surface tension with 'surface free energy' without any qualification will lead to the perpetuation of a misunderstanding which one had hoped had been eliminated years ago.

This book seems to have been constructed around a comprehensive set of lecture notes. General statements, a certain amount of hand-waving, and the occasional quip, may be justified in a lecture, when backed up by a reading list. But in a textbook one expects a much greater degree of precision, which is so essential in such a complex subject as colloid science. The misunderstandings which could arise from an uncritical reading of this book would be clarified by judicious use of the extensive references, but it is a great pity that a more rigorous approach has not been adopted, and that the underlying principles of colloid science do not shine through as clearly as they should. It is with considerable regret that I find myself unable to give this book my unqualified commendation.

D.H. Everett is Emeritus Professor of Physical Chemistry at the University of Bristol. 